\title{
Performance of UWB Communication based on Dynamic Bandwidth Direct Sequence
}

\author{
Jie Zhao \\ University of Wollongong \\ Wollongong, Australia
}

\author{
Stephen Culver \\ NokiaSiemens Networks \\ Helsinki, Finland
}

\author{
Mikael Gidlund \\ ABB AB, Corporate Research \\ Västerås, Sweden
}

\begin{abstract}
Ultra-wideband (UWB) communication is currently considered as a key technology of the next generation wireless personal area network (WPAN) and wireless local area network (WLAN). UWB operation do not require a spectrum license but UWB devices are required to share spectrum with licensed narrow band users. This paper presents a novel system for direct sequence (DS) UWB and the proposed dynamic bandwidth direct sequence (DBDS) system focuses on exploring a new idea rather than concentrating on impulse manipulation, provides a cognitive solution for DS-based UWB in a much simpler and more efficient way. The proposed system is able to transfer data under a fraction of original spread spectrum signal bandwidth and different spectral shapes while maintaining the same data rate. The system does not require generating specific impulse for working environment, therefore significantly reduces the system complexity. Simulation results demonstrate that the DBDS has a very exciting performance. Even received with different bandwidths and different spectral shapes, the data information can still be fully recovered at the same data rate.
\end{abstract}

\section{INTRODUCTION}

The exponential growth of wireless communication motivates the development of faster wireless devices not only for mobile or wide area network (WAN) but also for personal area network (PAN), and local area network (LAN). With the development of ultra-wideband (UWB) [1], transmission capacity has been increasing either through direct sequence (DS) [2], [3] or multi-band orthogonal frequency division multiplexing (MB-OFDM) UWB [4], [5], [6]. UWB technology provides high speed yet low power transmission through the occupation of a wide spectrum. However, coexistence issues between multiple UWB devices or between UWB and narrow band devices is serious challenge [7], [8]. One potential method to solve this problem is the use of cognitive radio over UWB technology that allows spectrum sharing and interference suppression among UWB devices.

DS-based UWB increases system performance over traditional impulse-based UWB by using DS spread spectrum technology, while maintaining a very simple structure. In DSbased UWB, each pulse occupies a wide spectrum bandwidth. Current spectrum sharing and coexistence solutions over DSbased UWB mainly focused on pulse shaping or how to generate a specific pulse that satisfy spectrum needs [9]. Special pulse generation requires complicated algorithms, not only increasing complexity but also being challenges for practical electronic circuits [10].

CHINACOM 2010, August 25-27, Beijing, China

Copyright $\odot 2011$ ICST 973-963-9799-97-4

DOI 10.4108/chinacom.2010.20
MB-OFDM has considered the issue of cognition from the very beginning of its design [11], [12]. This technology employs the concept of using traditional OFDM technology over multi-band. It can easily achieve spectrum sharing by simply turn off some transmit sub-bands that interferes with other devices. However, OFDM requires a large amount of processing such as FFT/IFFT that significantly increases the system complexity as well as power consumption.

\section{Contributions:}

- We develop a simple and efficient mechanism to adjust transmission bandwidth for direct sequence technology without jeopardizing the transmission rate. Filtering a symbol sequence with different filters according to the channel environment is the simplest way to adjust transmission bandwidth. Instead of reducing transmission rate as other traditional methods to avoid superimposition between chips, the new mechanism can keep the same data transfer speed.

- We propose a new cognitive radio system called dynamic bandwidth direct sequence (DBDS) for use in DS-based UWB systems. This systems enables UWB devices to transmit in $1 / 2,1 / 4,1 / 8$ or even less of the original bandwidth but keeps the same data rate.

Outline The reminder of the article is organized as follows. In Section II we discuss the system model and briefly review the. Section III introduces the proposed dynamic bandwidth direct sequence system and Section IV discuss obtained simulation results. Finally in Section V we conclude our work.

\section{System Model}

In order to focus on investigating the possibility to recover the signal through partial bandwidth, the wireless channel is assumed to be a slow Gaussian fading channel and the received signal can be expressed as

$$
r(t)=x(t) h(t)+z(t)
$$

where $x(t)$ is the transmitted signal, $h(t)$ is the channel response, and $z(t)$ is defined as the additive white Gaussian noise (AWGN) with double sided power spectral density $N_{0} / 2$. At the transmitter, the traditional multicode interleaved sequence forms a block of user data $a_{0}, a_{1}, \ldots, a_{N-1}$ with bit duration $T_{b}$ and $a_{i}$ can be expressed as following: 


$$
\begin{aligned}
& \sum_{i=0}^{N-1} \sum_{j=0}^{P-1} b_{i, j} g\left(t-i T_{c}-j N T_{c}\right) \\
= & \sum_{i=0}^{N-1} a_{i} \sum_{j=0}^{P-1} c_{i}\left[(j)_{p}\right] g\left(t-i T_{c}-j N T_{c}\right),
\end{aligned}
$$

where $c_{i}\left[(j)_{p}\right]$ is the orthogonal spreading sequence with period $P, c_{i_{[(j)]}}=1, j=0,1, \ldots, P-1$ is one period of this periodic sequence with duration $T_{c}$ and $[j]_{p}$ denotes $j$ modulo $P, g(t)$ is defined as a unit amplitude pulse which is zero outside the interval $\left[0, T_{c}\right]$. Within one period of multicode interleaving, $N$ bits of user data are spread with $P$ bits of orthogonal code for each bit of data. In an ideal Gaussian channel there are overall $N \times P$ chips produced during a period of multicode interleaving.

In an ideal Gaussian channel, this sequence is directly shaped by a filter which adjusts chip bandwidth and spread information of each chip onto nearby chips. This filter can be set to $1 / 2,1 / 4,1 / 8$ or even less of the original sequence bandwidth, that is, after the filter, output sequence bandwidth is shaped into $2^{m}$ of the original bandwidth. At the receiver end, chips are also processed in $N \times P$ chip block together. The received signals are firstly sampled with sampling period $T_{c}$, then passed through a matched filter. Filters at the TX and RX ends together achieve the spectrum shaping purpose. Since those chips are still interleaved and each chip has been overlapped into nearby $2^{m}$ chips, the filtered chip sequence in the receiver can be described as:

$r(n)=\sum_{i=0}^{N-1} \sum_{j=-P_{0}}^{P-1} \alpha_{i, j} \sum_{k=i-2^{m}}^{i+2^{m}-1} a_{k} c_{k}\left[(j)_{p}\right] g\left[(n-i-j N) T_{c}\right]+z_{n}$,

where $r(n)$ is the $n^{\text {th }}$ received sequence, $z_{n}$ is the white discrete-time Gaussian noise, $\alpha_{i, j}$ are tap coefficients that related to the filter and channel response. Since the wireless is assumed to be slow fading channel, $\alpha_{i, j}$ can be treated as constants. Note that $a_{-1} c_{-1}\left[(j)_{p}\right]=a_{k-1} c_{k-1}\left[(j)_{p}\right]$.

The despreading operation in this article is the same as in the original MCIDS system [6]. Received chips are filled columnwisely into $N \times P$ matrix. In order to perform despreading in the original MCIDS system, chips are simply read out in row-wise. However, in our proposed system, the chips are kept in the matrix for further processing. The remainder of $N \times P$ matrix is denoted

$$
E_{i, j}=\sum_{k=i-2^{m}}^{i+2^{m}-1} a_{k} c_{k}\left[(j)_{p}\right]
$$

Each received chip contains information of nearby $2^{m}$ original chips. Therefore only $N / 2^{m}$ chips are required to recover all transmitted data. Thus, after down-sampling the size of the matrix is $N / 2 \times P$, and each sampled element in the matrix can be expressed as

$$
r(l+1, j+1)=\alpha_{2^{*} l, j} \sum_{k=2^{*}(l-1)}^{2^{m}(l+1)-1} a_{k} c_{k}\left[(j)_{p}\right]+z_{2^{m} l, j},
$$

where $l=0,1,2, \ldots 2^{-m} N-1 ; j=0,1,2, \ldots P-1$. For detection of each bit $a_{i}$ is despeading, mathematically each row of the matrix is cyclically correlated with $P \times N$ bit long despread code $d_{i}[n]$. The despreading code is defined as

$d_{i}(n)=\sum_{j=0}^{P-1} c_{i}[j] \delta[n-i-j N], i=0, \ldots, N-1 . n=0, \ldots, P N-1$

If we also put despreading code into $P \times N$ matrix, then the despreading operation can actually be performed as matrix multiplication of the $N / 2^{m} \times P$ down-sampled receive-code matrix and the $P \times N$ despreading code matrix. Despreaded signals $U_{i, j}$ of code $d_{i}[n]$ at row $l$ are overlapped components with contributions from nearby chips. Mathematically, we have

$$
U_{i, l}=\sum_{j=0}^{P-1} r(l+1, j+1) c_{i}\left[(j)_{p}\right]
$$

Suppose that $U_{i}$ is the decision variable and this variable is obtained by the correlation of the despread signal $U_{i, l}$ and the configuration of the corresponding tap coefficients $a_{l, j}^{*}$. To collect energy from all $l$ chips, we have

$$
\begin{aligned}
U_{i} & =\operatorname{Re}\left[\sum_{l=1}^{2^{-m} N} U_{i, l} \alpha_{2^{m} l, j}^{*}\right] \\
& =\operatorname{Re} \sum_{l=1}^{2^{-m} N} \sum_{j=0}^{P-1} \alpha_{2^{m} l, j}^{*} \alpha_{2^{m} l, j} \sum_{k=2^{m}(l-1)}^{2^{m}(l+1)-1} a_{k} c_{k}\left[(j)_{p}\right] \\
& +\operatorname{Re} \sum_{l=1}^{2^{-m} N} \alpha_{2^{m} l, j}^{*} \sum_{j=0}^{P-1} z_{2^{m} l, j}
\end{aligned}
$$

Since $c_{i}\left[(j)_{p}\right]$ are orthogonal sequences, the satisfy

$$
\sum_{k=2^{m}(l-1)}^{2^{m}(l+1)-1} a_{k} c_{k}\left[(j)_{P}\right] c_{P}\left[(j)_{P}\right]=
$$

When $i=k=l$, that is when the spreading code matches the despreading codes, the decision variable $U_{i}$ becomes

$$
U_{i}=P \sum_{l=1}^{2^{-m}} \sum_{j=0}^{P-1}\left|\alpha_{2^{m} l, j}\right|^{2} a_{i}+\operatorname{Re} \sum_{l=1}^{2^{-m}} \sum_{j=0}^{N-1} \alpha_{2^{m} l, j}^{*} z_{2^{m} l, j}
$$

From this we can observe the following. The user data will only be received when the corresponding spreading code and despreading code are matched. In this situation, the final decision variable $U_{i}$ will be an amplified value of original user data bit plus Gaussian noise. Otherwise, when spreading and 


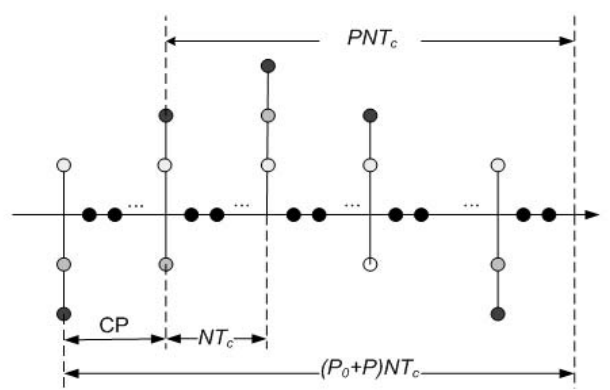

Fig. 1. Every $2^{m}$ chips are added into one chip, while others are set to zero.

despreading code are not matched, despread data will be zero with just Gaussian noise, and no other data bit that is spread or despread by other code is involved.

\section{Proposed Algorithm for Multipath Fading}

In multipath channel, signals through different paths arrive with different delays and thus cause ISI. The proposed system spread $N$ bit user data with $P$ bit code for each data, and processes $N \times P$ chips at a time. The output interleaved sequence can be recognized as $P$ vectors with $N$ chips in each vector. At the $\mathrm{RX}$ end, $P$ vectors are also processed together and user data are collected from every single vector. Due to the fact that transmitted chips are interleaved, ISI between different received vectors can cause major distortion in output chips after deinterleaving and therefore significantly reduce system performance. ISI will make filtered chips in every vector completely messed up which makes the determination of channel estimation coefficients from the received symbols extremely difficult. To solve the ISI problem we rely on the symbol combination operation at the TX end. The combining operation row wisely merges element of every $2^{m}$ rows into one row, but retain the same matrix size. The elements in the first of every $2^{m}$ row are replaced by the respective sums of all $2^{m}$ rows, and the remaining elements are set to zero. As Fig. 1 shows, $2^{m}$ chips are combined into one chip, thus those $2^{m}$ chips will take the same amount of fading as well as filtering at the same time. Therefore, the tap coefficients that relate to filter and channel response for those $2^{m}$ original symbols will have the same value. This means that detecting tap coefficient can be as easy in an ideal Gaussian channel. Assuming that the total multipath delay are less than $2^{m}$ chip duration. If we take $G_{n}=\left(a_{n}+b_{n}+c_{n}\right)$, and $G_{n}^{m}$ is the received
$G_{n}$ from path $m$ and the received sequence is given by $\left\{G_{0}^{0}, \ldots, G_{0}^{m-1}, G_{1}^{0}, \ldots, G_{1}^{m-1}, \ldots, G_{n-1}^{0} G_{n-1}^{1}, \ldots, G_{n-1}^{m-1}\right\}$. This combined sequence also brings a benefit that system performance can be improved by collecting all the energy of the chips from multiple paths. That is why simple sampling operation at the RX end is replaced by cyclic sampling ${ }^{1}$.

An easy way to achieve MCIDS with CP is to put the spread symbols in row-wise into a matrix with $N \times P$ rows and $P$ rows, where the first $P_{0}$ row is $\mathrm{CP}$, the output symbols in column-wise. We assume the channel is multipath fading with Gaussian noise and that no more than $2^{m}-1$ total paths, the received signal can generally be expressed as in (4.1). Interleaved chips are also column-wisely filled into $\left(N+P_{0}\right) \times P$ matrix, where $N$ is the $N^{t h}$ data bit. Then, all the $P_{0}$ rows of CPs are removed to suppress ISI from different vector. Take $G_{i, j}=\sum_{k=i+2^{2}-1}^{(i+1) 2^{m}-1} a_{k} c_{k}\left[(j)_{p}\right]$ as the reordered chip, where $i=0,1, \ldots,(N-1) / 2^{m}, m=0,1, \cdots$ and $E_{i, j}=\sum_{k=1-2^{m}}^{i+2^{m}-1} G_{k, j}$ indicates that $2^{m}$ of nearby chips are overlapped by filtering.

It is clear that each received chip contains information of nearby $2^{m}$ original samples, and every $2^{m}$ chip in column contains the same user bit. Therefore, only $N / 2^{m}$ chips are required to recover all transmitted data. However, collecting energy paths by cyclic sampling can improve system performance. Each element in the $(k+1)^{t h}$ row and the $(j+1)^{t h}$ column in the down sampled matrix can be expressed as

$$
r(k+1, j+1)=\alpha_{2^{k} k, j} E_{k, j}+z_{2^{m} k, j},
$$

where $l=1,1,2, \ldots, 2^{-m} N-1 ; j=0,1, \ldots P-1$. As chips from different path contain the same information as the original, the cyclic down sampled elements from different paths are just repeats of the same information. Therefore, we can use one group of the sampled matrix as a demonstration of the algorithm. The processing applies to the sampled matrix which is collected from other paths.

The despreading operation performs as a matrix multiplication for the down-sampled matrix and the despreading vector.

$$
U_{i, l}=\sum_{j=0}^{P-1} r(l+1, j+1) c_{i}\left[(j)_{p}\right] .
$$

Suppose that $U_{i}$ is the decision variable which is obtained by correlation of despread signal $U_{i, l}$ and the conjugate of corresponding tap coefficients $\alpha_{l, j}^{*}$. To collect energy from all $l$ chips, we have

\footnotetext{
${ }^{1}$ For example, we first sample $\left\{G_{0}^{0}, G_{0}^{1} \ldots G_{0}^{m-1}\right\}$, then $\left\{G_{1}^{0}, G_{1}^{1} \ldots G_{1}^{m-1}\right\}$, all over to $\left\{G_{n-1}^{0}, G_{n-1}^{1} \ldots G_{n-1}^{m-1}\right\}$
} 


$$
\begin{aligned}
U_{i} & =\operatorname{Re}\left[\sum_{l=1}^{2^{-m} N} U_{i, j} \alpha_{2^{m} l, j}^{*}\right] \\
& =\operatorname{Re}\left[\sum_{l=1}^{2^{-m}} \sum_{j=0}^{P-1}\left(\alpha_{2^{m} l, j} E_{i, j}+z_{2^{m} l, j}\right) \alpha_{2^{m} l, j}^{*}\right] \\
& =\operatorname{Re} \sum_{l=1}^{2^{-m} N} \sum_{j=0}^{P-1} \alpha_{2^{m} l, j}^{*} \alpha_{2^{m} l, j} \\
& \times \sum_{i=n-2^{m}}^{m} \sum_{k=2^{m} l-1}^{2^{m}(l+1)-1} a_{k} c_{k}\left[\left(j_{P}\right)\right] c_{i}\left[(j)_{P}\right] \\
& +\operatorname{Re} \sum_{l=1}^{2^{-m}} \sum_{j=0}^{P-1} \alpha_{2^{m} l, j}^{*} z_{2^{m} l, j}
\end{aligned}
$$

Since $c_{i}\left[(j)_{P}\right]$ are orthogonal sequences, they satisfy

$$
\sum_{i=n-2^{m}}^{n+2^{m}-1} \sum_{k=2^{m} l-1}^{2^{m}} c_{k}\left[(j)_{P}\right] c_{i}\left[(j)_{P}\right]= \begin{cases}P, & i=k=1 \\ 0, & \text { otherwise }\end{cases}
$$

When $i=k=l=n$, that is, when the corresponding spreading code and despreading code match, the decision variable $U_{i}$ becomes

$$
U_{i}=P \sum_{l=1}^{2^{-m}} \sum_{j=0}^{N-1}\left|\alpha_{2^{m} l, j}\right|^{2} a_{i}+\operatorname{Re} \sum_{l=1}^{2^{-m}} \sum_{j=0}^{P-1} \alpha_{2^{m} i, j}^{*} z_{2^{m} i, j}
$$

Even thought the ISI effect causes serious disorder in the received chips, user data can still be used when the corresponding spreading code and despreading code match each other.

\section{NUMERICAL RESUltS}

To assess the performance of the proposed system we consider simulations.In this article we have used BPSK modulation and the user data period is set to $4 \times 10^{-8}$ seconds. The cyclic prefix length is 4 bit and is expected to cause 2$3 \mathrm{~dB} E_{b} / N_{0}$ loss. We consider simulations under both ideal Gaussian channel and multipath channel.

Ideal Gaussian Channel In this case the bandwidth adjustment rate can be set to either $1 / 2$ and $1 / 4$. We also consider different filter types, namely, raised cosine filter, high pass filter and band pass filter.

Figure 2 show the BER results of an ideal Gaussian channel and it can be seen that by limiting the transmission bandwidth into $1 / 2$ or $1 / 4$ of the original bandwidth does not harm bit error rates of the DBDS system. We also observe that the proposed system matches the theoretical results which strongly indicates that DBDS is a lossless transmission algorithm. In order to suppress ISI, we have appended a 4 bit CP to the system and that will include a $2 \mathrm{~dB}$ loss in system performance as shown in Fig. 3
Multipath Gaussian Channel Figure 4 show the BER performance under multiple Gaussian channel. The obtained results show that the system performance remains stable under different transmission bandwidths different shapes of spectrum as long as the multipath delay is less than the minimal value if $\mathrm{CP}$ duration and $2^{m}$ chip duration, if transmission bandwidth is limited to $2^{m}$ of the original bandwidth.

We can make the following conclusion from the obtained simulation results. In an ideal Gaussian channel, the DBDS system offers a lossless solution for cognitive UWB. Its BER curves math the theoretical results even though transmission bandwidth already have been limited by $1 / 2$ and $1 / 4$ of original. and the only performance loss is due to the insertion of CP.

In the case of multipath Gaussian channel, the DBDS system combines every $2^{m}$ of chips into one in between interleaving and filtering step. This operation forces $2^{m}$ interleaved symbols to face the same level of variation during transmission. The performance loss is due to the difficulty in detecting tap coefficients for each symbol is overcome. The DBDS system under multipath channel provides performance close to that in an ideal Gaussian channel.

Compared to traditional DS-based UWB transmission, the DBDS system can fully recover user data from different transmission bandwidth. Furthermore, according to the achieved simulation results under different channels, different transmission, and different spectrum shaping filters, the DBDS system offers the same BER as the full bandwidth transmission. Due to this advantage, the system enables multiple UWB devices to share the same spectrum. For example, one uses lower half of the available spectrum, and another uses the upper half, but none of them will have data rate lost or interference between them.

\section{Conclusions}

In this article, we propose and investigate a dynamic bandwidth direct sequence (DBDS) UWB system as a novel cognitive solution for direct sequence (DS) UWB. Since current cognitive solutions for DS-based UWB mainly focuses on pulse generation in order to produce a specific impulse according to the working spectrum environment and this include a large mount of calculations while the proposed DBDS scheme do not require specific impulse generation to dynamically adjust transmission bandwidth.

Utilizing multi-code interleaving, symbol combining, bandwidth filtering and cyclic down sampling, the proposed system is able to dynamically compress the transmission into $1 / 2,1 / 4,1 / 8$ or even less of the original bandwidth without decreasing its data rate. The proposed system can fully recover signals at different transmission bandwidth and different transmission band, only slightly increasing the systems complexity compared to a full bandwidth system.

\section{REFERENCES}

[1] M. Bettayeb and S. F. A. Shah, "State-of-the-art ultra-wideband technology for communication systems: a review," in Proc. 10th IEEE Inter- 


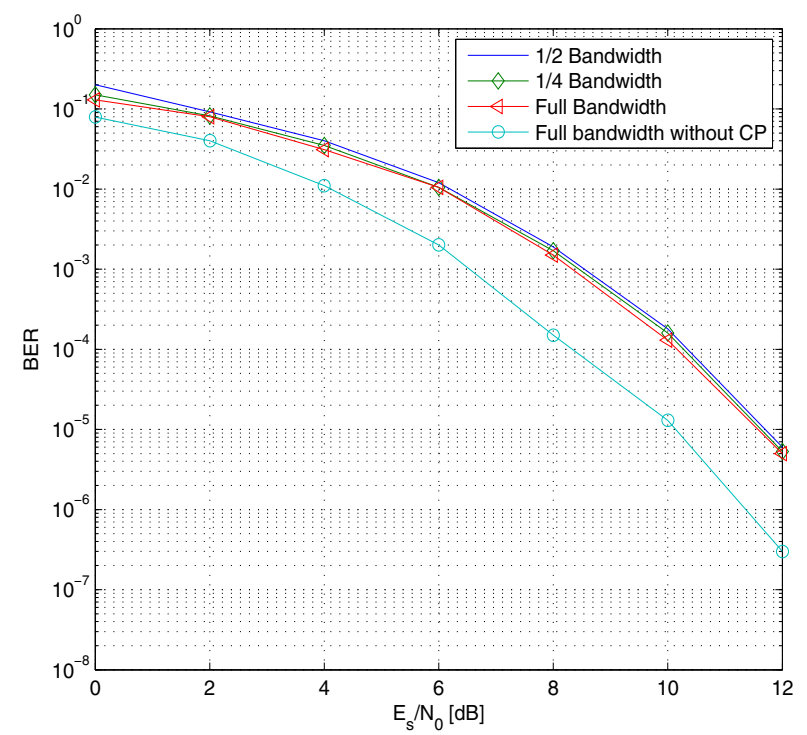

Fig. 2. Average performance using raised cosine filter with 4 bit $\mathrm{CP}$ in ideal Gaussian channel.

national Conference on Electronics, Circuits and Systems (ICECS'03), 2003.

[2] S. Franz, and U. Mitra, "On optimal data detection for UWB transmitted reference system," in Proc. IEEE Global Communications Conference (GLOBECOM'03), San Fransisco, USA, Dec. 2003.

[3] Y. Soulimi and R. Knopp, "On the achievable rates of ultra-wideband PPM with non-coherent detection in multipath environments," in Proc. IEEE International Conference on Communications (ICC'03), Anchorage, Alska, USA, May 2003.

[4] A. Batra, S. Lingam, and J. Balakrishnan, "Multi-band OFDM: a cognitive radio for UWB," in Proc. International Symposium on Circuits and Systems, (ISCS'06), 2006.

[5] A. Batra, J. Balakrishnan, G. Aiello, J. Foerster and A. Dabak, "Design of a multiband OFDM system for realistic UWB channel environments," IEEE Trans. on Microwave Theory and Tech., vol. 52, no. 9, pp. 21232138 , Sept. 2004

[6] H. Yamaguchi, "Active interference cancellation technique for MBOFDM cognitive radio," in Proc. 34th European Microwave Conference, 2004.

[7] M. Lai, and S. K. Jeng, "A microstrip three-port and four-channel multiplexer for WLAN and UWB coexistence," IEEE Trans. on Microw. Theory and Techn., vol. 53, pp. 3244-3240, 2005.

[8] P. He, Y. Lu, H, Zhang, and J. Lu, "A pulse shaping method for UWB avoding the frequency coexistence interference with WLAN," in Proc. 2nd International Conference on Mobile Technology, Applications and Systems," 2005.

[9] L. Huang, H. X. Zhang, and Y. H. Lu, "A novel method to generate UWB shaping pulses based on chip pulse," in Proc. 6th International Conference on ITS Telecommunications, 2006.

[10] W. Zhang, H. Shen, and K. S. Kwak, "Pulse series based UWB CR system," in Proc. International Workshop on Cross Layer Design, 2007.

[11] X Zhou, K. Y. Yazdandoost, H. Zhang and I. Chlamtac, "Cognospectrum: spectrum adaptation and evolution in cognitive ultra-wideband radio," in Proc. IEEE International Conference on Ultra-Wideband, 2005.

[12] F. Xu, W. Zhong, and Z. Zhou, "Cognitive Interference suppress in UWB system using modified Hermite polynomials pulse," in Proc. Communications and Information Technologies, 2006.

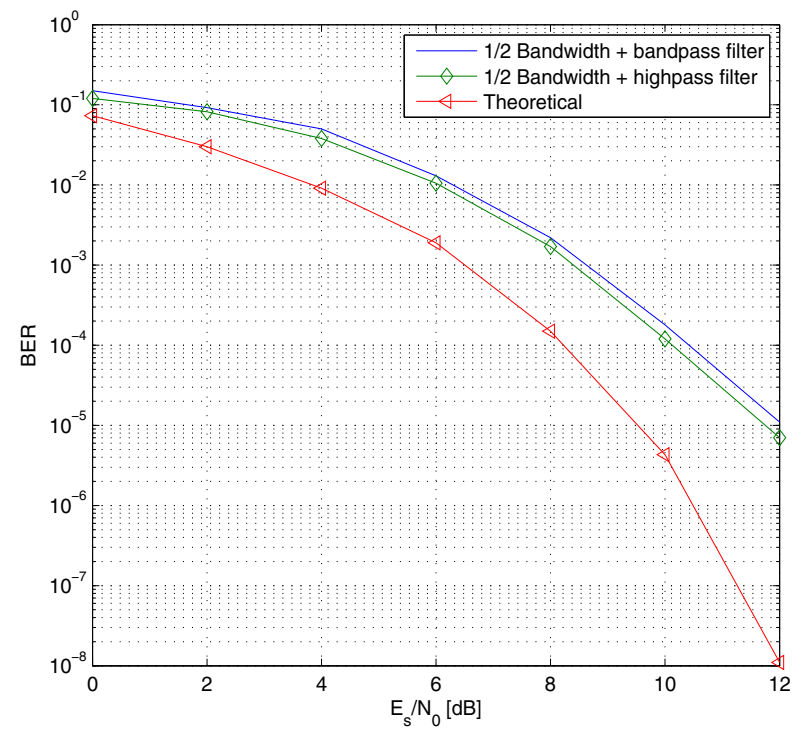

Fig. 3. Average performance using half bandwidth high pass and low pass filter with 4 bit CP in ideal Gaussian channel.

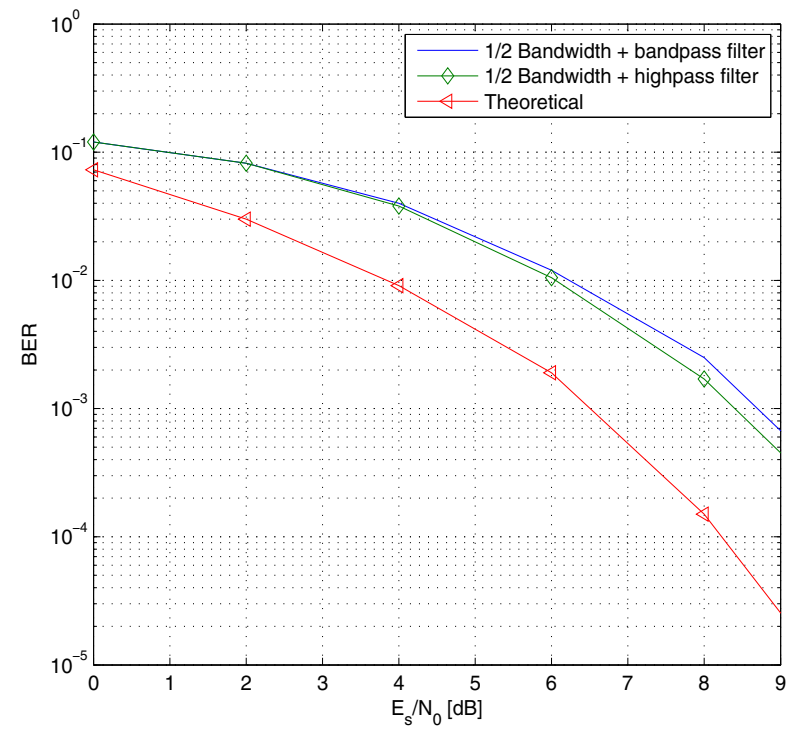

Fig. 4. Average performance by half bandwidth (a) band pass and (b) high pass filter under multipath Gaussian fading. 